\title{
Equilibrium Equity Premium in a Semi Martingale Market When Jump Amplitudes Follow a Binomial Distribution
}

\author{
George M. Mukupa ${ }^{1}$, Elias R. Offen ${ }^{2}$ \\ Zambia \\ ${ }^{2}$ Department of Mathematics, University of Botswana, Gaborone, Botswana \\ Email: gmukupa@mu.ac.zm,offen@mopipi.ub.bw
}

${ }^{1}$ School of Science, Engineering and Technology, Department of Mathematics and Statistics, Mulungushi University, Kabwe,

How to cite this paper: Mukupa, G.M. and Offen, E.R. (2018) Equilibrium Equity Premium in a Semi Martingale Market When Jump Amplitudes Follow a Binomial Distribution. Journal of Mathematical Finance, 8, 599-612.

https://doi.org/10.4236/jmf.2018.83038

Received: May 16, 2018

Accepted: August 17, 2018

Published: August 20, 2018

Copyright $\odot 2018$ by authors and Scientific Research Publishing Inc. This work is licensed under the Creative Commons Attribution International License (CC BY 4.0).

http://creativecommons.org/licenses/by/4.0/

\begin{abstract}
This paper studies equilibrium equity premium in a semi martingale market when jump amplitudes follow a binomial distribution. We take $n$ to be the number of times. An investor is trading in this market with $p$ being the probability that there is a shift in the price at the trading time $t$. We find significant variations in the equilibrium equity premium for the martingale and semi martingale markets in terms of wealth value, volatility and other parameters under study. In this market, the equilibrium equity premium remains constant regardless of volatility and wealth value.
\end{abstract}

\section{Keywords}

Binomial Distribution, Semi Martingale, Risk Premium, Jump Diffusion

\section{Introduction}

A semi martingale market is a partially predictable market with a decomposition

$$
X_{t}=X_{0}+M+A \text {, }
$$

such that $M=\left(M_{t}\right)_{0 \leq t \leq T}$ is a square-integrable martingale with $M_{0}=0$ and $A=\left(A_{t}\right)_{0 \leq t \leq T}$ being a predictable process of finite variation $|A|$ with $A_{0}=0$. This market is so attractive to investors as it is deemed fair and enables uncertainity risks to be compensated fairly. This fair compensation is usually termed as Risk Premium which of late has attracted a lot of attention from researchers. [1] and [2] had considered cases where this premium evolves according to a compensated compound poisson process in the martingale 
market, but to make it more interesting, our paper considers the evolution of the model in the semi martingale market by fixing the jump amplitudes to follow a Binomial distribution.

[3] modeled a stock price as a production process in a production economy with jump diffusion and established a general equilibrium model for the equity premium. These authors proposed a pricing kernel and used it to price options. Specifically, [3] derived analytical expressions for the return distributions in the physical and the risk-neutral measures, and their model provided empirical evidence supporting the negative risk-neutral skewness and the relation between the moments of the risk-neutral and physical distributions. Their model provided more empirical evidence supporting the negative excess return of a Delta-hedged portfolio.

More recent studies [4] [5] [6] and [7] have developed an equilibrium asset and option pricing model in a production economy under jump diffusion. Their model was based on the intertemporal general equilibrium model of a production economy. Asset pricing in [8] provided analytical formulas for an equity premium and a more general pricing kernel that links the physical and risk neutral densities, which explained the two empirical phenomena of the negative variance risk premium and implied volatility smirk when a market crash is expected. [8] showed that jump size was indeed negative and that the risk aversion coefficient assumed a reasonable value when taking the jump into account. However, despite studying the systematic risk premium of the market portfolio, [8] did not model the impact of new technological developments and the dynamics of individual stock prices.

A discrete random variable (RV) is a Binomial if it arises from Bernoulli trials. There is a fixed number, $n$, of independent trials which by independence, means the result of any trial (for example, trial 1) does not affect the results of the following trials, and all trials are conducted under the same conditions. Under these circumstances, we define the binomial random variable $X$ as the number of successes in $n$ trials. The notation $X \sim B(n, p)$ is usually used to imply that $X$ is a random variable following a binomial distribution. The mean is $\mu=n p$ and the standard deviation is $\sigma=\sqrt{n p q}$ for some probability of failure $q$. The probability of exactly $x$ successes in $n$ trials is $p$. In this market, everytime we observe a jump (shift in price), we record it as a success with probability of occurence, $p$. Therefore, $p=E\left(\mathrm{~d} N_{t}\right)=\lambda \mathrm{d} t$ which studies by [9] and [10] took as the probability that $N_{t}$ will jump once in the given period.

In literature, Jump Diffusion has been widely used in Option Valuation as opposed to modelling equity premium. In discrete time, the theory was proposed by [11] and later developed by [3] [4] [5] [6] [7] [12]-[17] where the Binomial option pricing model has been extensively used. More recent studies [4] [5] [6] [7] [15] [16] and [17] have developed an equilibrium asset and option pricing model in a production economy under jump diffusion. For general cases, one can see [18]-[28]. In our case, we use the binomial principles in finance to model equilibrium equity premium when the price process is a semi martingale. 
Unlike [9] who fixed $P$ to positive jumps, we take this probability whenever we observe a jump (whether positive or negative). This allows us to study the impact of jumps themselves on the equity premium.

Our contribution in this paper is comparable to [3] and also further elaboration by [9] and [10] who considered the martingale case of equilibrium equity premium. This contribution enables investors to compare the martingale and semi martingale markets in terms of the premium expected for having taken some risk in the equity market. This will make them be aware of the necessary judgements to be undertaken before one can consider investing in a partly predictable market whose price process can either go up or come down in value.

\section{The Model}

To formulate the model, we consider that the price process is arising from a Binomial distribution with one parameter $p$, the probability of observing a jump in a given time interval $[0, t)$ and since the market is partially volatile, our process will evolve as a compensated compound poisson process similar to that of [3] and also further elaboration by [9] and [10].

Let's consider a Jump Diffusion process;

$$
\mathrm{d} X_{t}=\mu \mathrm{d} t+\delta \mathrm{d} B_{t}+\left(\mathrm{e}^{x}-1\right) \mathrm{d} N_{t}-\lambda E\left(\mathrm{e}^{x}-1\right) \mathrm{d} t .
$$

which is a semi martingale with discontinuities because of the presence of jumps.

We take $\mu, \delta$ and $\lambda$ as constants and $x$ as a vector of jump sizes following a binomial distribution. The processes $B_{t}$ and $N_{t}$ are independent. This follows directly from the definition of Brownian motion as being a continuous process and the Poisson being discrete which we obviously know that continuous processes and discrete are independent. $\lambda$ is the frequency of the Poisson process. We set $\left(\mathrm{e}^{x}-1\right)$ in the jump process so that $\mathrm{e}^{x}-1=0$ if there is no jump as $x$ is then a zero vector. $E$ is the expectation which makes the process $\mathrm{e}^{x}-1$ deterministic. $\mathrm{d} N_{t}$ models the sudden changes as a result of rare events happening and $\mathrm{d} B_{t}$ models small continuous changes generated by the noise whose volatility is a constant $\delta$.

The compensated compound Poisson process $\left(\mathrm{e}^{x}-1\right) \mathrm{d} N_{t}-\lambda E\left(\mathrm{e}^{x}-1\right) \mathrm{d} t$ has the mean of zero because

$$
E\left[\left(\mathrm{e}^{x}-1\right) \mathrm{d} N_{t}-\lambda E\left(\mathrm{e}^{x}-1\right) \mathrm{d} t\right]=E\left(\mathrm{e}^{x}-1\right) E\left(\mathrm{~d} N_{t}\right)-E\left(\mathrm{e}^{x}-1\right) E(\lambda \mathrm{d} t)=0
$$

and $E\left(\mathrm{~d} N_{t}\right)=\lambda \mathrm{d} t$.

To solve

$$
\mathrm{d} X_{t}=\mu \mathrm{d} t+\delta \mathrm{d} B_{t}+\left(\mathrm{e}^{x}-1\right) \mathrm{d} N_{t}-\lambda E\left(\mathrm{e}^{x}-1\right) \mathrm{d} t,
$$

we do not need to apply Itô Lemma with Jumps because the diffusion part is a continuous semi martingale whose procedure for solution does not require the integrating factor. We solve for the price process at the terminal time $T$ as follows: 


$$
\mathrm{d} X_{t}=\left[\mu-\lambda E\left(\mathrm{e}^{x}-1\right)\right] \mathrm{d} t+\delta \mathrm{d} B_{t}+\left(\mathrm{e}^{x}-1\right) \mathrm{d} N_{t}
$$

By integration we have

$$
X_{T}=X_{t}+\left[\mu-\lambda E\left(\mathrm{e}^{x}-1\right)\right] \tau+\delta B_{\tau}+\sum_{i=1}^{N_{\tau}}\left(\mathrm{e}^{x_{i}}-1\right), \text { for } \tau=T-t
$$

as the investment period.

Suppose also that, at the risk-free rate $\rho$, the money market account $X_{0}(t)$ is such that

$$
\mathrm{d} X_{0}(t)=\rho(t) X_{0}(t) \mathrm{d} t
$$

whose total supply is assumed to be zero. Consider here that $\rho$ is risk-free because it is the rate for the non risky asset (money account).

Since the value of someone's investment in this production economy at any time $t$ is given by $V_{t}=\phi X_{t}$, for some portfolio $\phi=(1-\omega, \omega)$ consisting of $1-\omega$ non risky assets and $\omega$ risky assets, we have that by the self financing strategy,

$$
\mathrm{d} V_{t}=\phi \mathrm{d} X_{t}
$$

so that the total wealth at any time $t$ is

$$
V_{t}=V_{0}(t)+V_{1}(t)
$$

where $V_{0}(t)$ is the value of the money market account and $V_{1}(t)$ is the value of the investment in the stock market at time $t$.

Now

$$
\begin{aligned}
\mathrm{d} V_{t} & =\mathrm{d} V_{0}(t)+\mathrm{d} V_{1}(t)=(1-\omega) \mathrm{d} X_{0}(t)+\omega \mathrm{d} X(t) \\
& =(1-\omega)\left(\rho X_{0}(t) \mathrm{d} t\right)+\omega\left[\mu-\lambda E\left(\mathrm{e}^{x}-1\right)\right] \mathrm{d} t+\omega \delta \mathrm{d} B_{t}+\omega\left(\mathrm{e}^{x}-1\right) \mathrm{d} N_{t} .
\end{aligned}
$$

Since the equity premium $\phi=\mu-\rho$, we have that $\mu=\phi+\rho$, hence

$$
\begin{aligned}
\mathrm{d} V_{t}= & {\left[\rho X_{0}(t)-\omega \rho X_{0}(t)+\omega \phi+\omega \rho-\lambda \omega E\left(\mathrm{e}^{x}-1\right)\right] \mathrm{d} t } \\
& +\omega \delta \mathrm{d} B_{t}+\omega\left(\mathrm{e}^{x}-1\right) \mathrm{d} N_{t} .
\end{aligned}
$$

The investor's optimal control problem then is to maximize his expected utility function

$$
\max E_{t} \int_{t}^{T} y(t) U\left(r_{t}\right) \mathrm{d} t
$$

subject to

$$
\begin{aligned}
\mathrm{d} V_{t}= & {\left[\rho X_{0}(t)-\omega \rho X_{0}(t)+\omega \phi+\omega \rho-\lambda \omega E\left(\mathrm{e}^{x}-1\right)-r_{t}\right] \mathrm{d} t } \\
& +\omega \delta \mathrm{d} B_{t}+\omega\left(\mathrm{e}^{x}-1\right) \mathrm{d} N_{t}
\end{aligned}
$$

The wealth ratio $\omega$ and consumption rate $r_{t}$ are control variable. The general equilibrium occur when $\omega=1$.

\section{Results and Discussion}

Theorem 1. In a semi martingale market with binomial jumps, an investor's 
equilibrium equity premium with CRRA power utility function $U\left(r_{t}\right)=\frac{r_{t}^{\beta}}{\beta}, 0<\beta<1$, in the production economy with jump diffusion is given by

$$
\begin{aligned}
\phi= & \rho X_{0}(t)-\rho-(\beta-1) V_{t}^{-1} \delta^{2}+\lambda(1+(\mathrm{e}-1) p)^{n} \\
& -\lambda q\left(1-p+p \mathrm{e}^{\beta}\right)^{n}-\lambda+\lambda q\left(1-p+p \mathrm{e}^{\beta-1}\right)^{n}
\end{aligned}
$$

where $\phi_{\delta}=\rho X_{0}(t)-\rho-(\beta-1) V_{t}^{-1} \delta^{2}$ is the diffusive risk premium and $\phi_{N}=\lambda(1+(\mathrm{e}-1) p)^{n}-\lambda q\left(1-p+p \mathrm{e}^{\beta}\right)^{n}-\lambda+\lambda q\left(1-p+p \mathrm{e}^{\beta-1}\right)^{n}$ is the rare-event premium.

Proof. If $X$ is a random variable with a binomial distribution, then $Y=\mathrm{e}^{X}$ is a logbinomial random variable.

In particular, if $X \sim B(n, p)$ and $Y=\mathrm{e}^{X}$ then $Y^{k}=\mathrm{e}^{k X}$. Also

$$
E\left[\mathrm{e}^{k X}\right]=m_{X}(k)
$$

where $m_{X}(k)$ is the moment-generating function of $X$ evaluated at $k$. Hence

$$
E\left[\mathrm{e}^{k X}\right]=\left(1-p+p \mathrm{e}^{k}\right)^{n}
$$

and so

$$
E\left[\mathrm{e}^{X}\right]=(1-p+p \mathrm{e})^{n}=(1+(\mathrm{e}-1) p)^{n}=m_{X}(1)
$$

Let $X=x$ be a vector of binomially distributed jump sizes then for the power utility function of [10], the rare-event premium $\phi_{N}=\lambda E\left[\left(\mathrm{e}^{x}-1\right)\left(1-V_{t}\left(\mathrm{e}^{x}\right)^{\beta-1}\right)\right]$ which becomes $\phi_{N}=\lambda E\left[\mathrm{e}^{x}-V_{t} \mathrm{e}^{x+x(\beta-1)}-1+V_{t} \mathrm{e}^{x(\beta-1)}\right]$.

Now, taking $E\left[V_{t}\right]=q$, we realise that:

$$
\begin{gathered}
E\left[\mathrm{e}^{x+x(\beta-1)}\right]=E\left[\mathrm{e}^{x(1+\beta-1)}\right]=E\left[\mathrm{e}^{\beta x}\right]=\left(1-p+p \mathrm{e}^{\beta}\right)^{n}=m_{X}(\beta) . \\
E\left[\mathrm{e}^{x(\beta-1)}\right]=\left(1-p+p \mathrm{e}^{\beta-1}\right)^{n}=m_{X}(\beta-1) .
\end{gathered}
$$

Therefore, our rare-event premium

$$
\phi_{N}=\lambda\left[E\left(\mathrm{e}^{x}\right)-q E\left(\mathrm{e}^{x+x(\beta-1)}\right)-1+q E\left(\mathrm{e}^{x(\beta-1)}\right)\right]
$$

now becomes

$$
\phi_{N}=\lambda\left[(1+(\mathrm{e}-1) p)^{n}-q\left(1-p+p \mathrm{e}^{\beta}\right)^{n}-1+q\left(1-p+p \mathrm{e}^{\beta-1}\right)^{n}\right]
$$

which implies that our equity premium is now

$$
\begin{aligned}
\phi= & \rho X_{0}(t)-\rho-(\beta-1) V_{t}^{-1} \delta^{2}+\lambda(1+(\mathrm{e}-1) p)^{n} \\
& -\lambda q\left(1-p+p \mathrm{e}^{\beta}\right)^{n}-\lambda+\lambda q\left(1-p+p \mathrm{e}^{\beta-1}\right)^{n} .
\end{aligned}
$$

Figure 1 suggests a constant equity premium regardless of how volatile the process becomes. This is a good result for investors in this market because they 


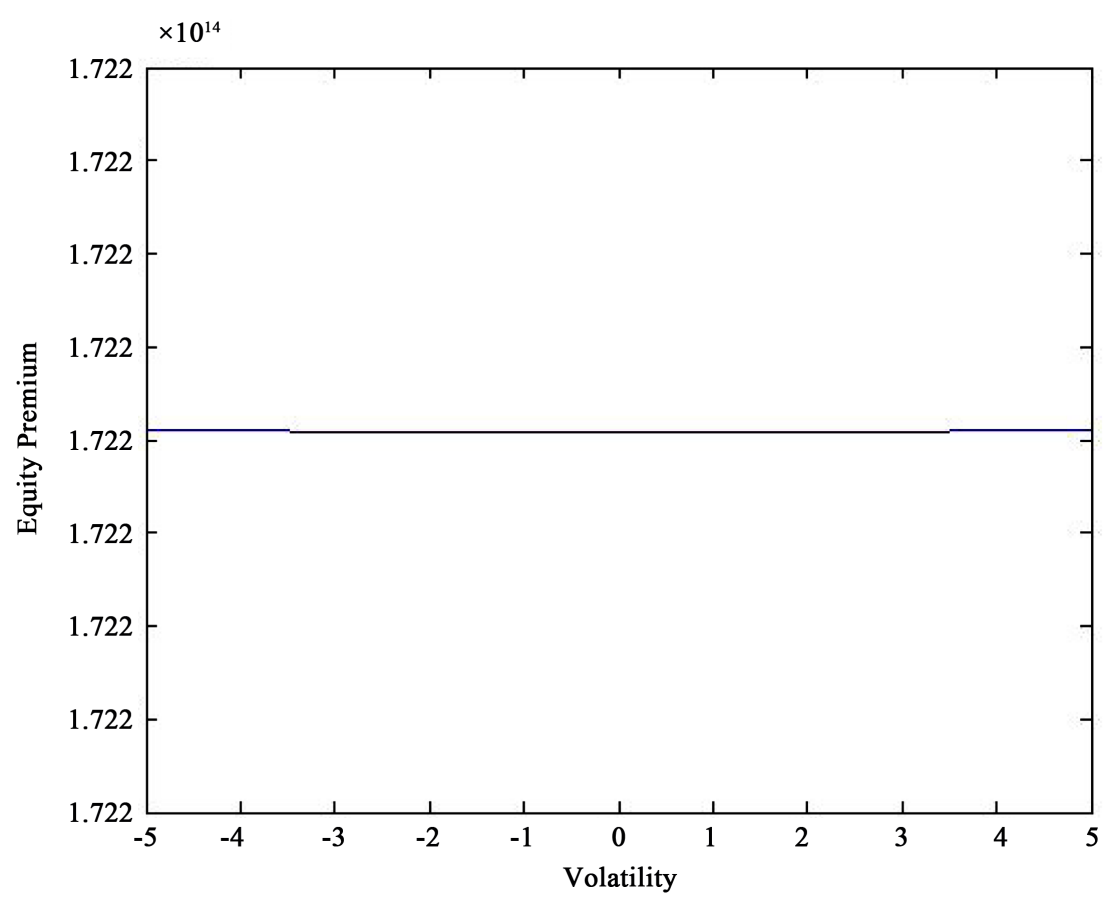

Figure 1. Power utility volatility effect under binomial distribution.

are assured of some compensation regardless of the fall or rise in the trading prices. Infact, when there is no jump expected, the premium is symmetrical about zero volatility and increases on either side (see Figure 2). This means that the diffusive risk premium always exist and is positive in this market. Therefore, the change in the price process due to time, is fairly compensated. In terms of Beta effect, it is clear from Figure 3 that the premium is zero whenever $\beta<2$ and decreases otherwise. This parameter affects the premium in an unattractive manner as it is inversely proportional to the equity premium on a large scale. This is confirmed in Figure 4 as we observe an inversely proportional relationship between the equity premium and beta. This is a case when jumps are not expected. However, when jumps are expected, the compensation is fair.

Theorem 2. In the semi martingale market with binomial jumps, the investo's equilibrium equity premium with square root utility function $U\left(r_{t}\right)=\sqrt{r_{t}}, r_{t}>0$ is given by

$$
\begin{aligned}
\phi= & \rho X_{0}(t)-\rho+\frac{\delta^{2}}{2 V_{t}}+\lambda(1+(\mathrm{e}-1) p)^{n} \\
& -\lambda q\left(1-p+p \mathrm{e}^{\frac{1}{2}}\right)^{n}-\lambda+\lambda q\left(1-p+p \mathrm{e}^{-\frac{1}{2}}\right)^{n}
\end{aligned}
$$

where $\phi_{\delta}=\rho X_{0}(t)-\rho+\frac{\delta^{2}}{2 V_{t}}$ is the diffusive risk premium and $\phi_{N}=\lambda(1+(\mathrm{e}-1) p)^{n}-\lambda q\left(1-p+p \mathrm{e}^{\frac{1}{2}}\right)^{n}-\lambda+\lambda q\left(1-p+p \mathrm{e}^{-\frac{1}{2}}\right)^{n}$ is the rare-event premium. 


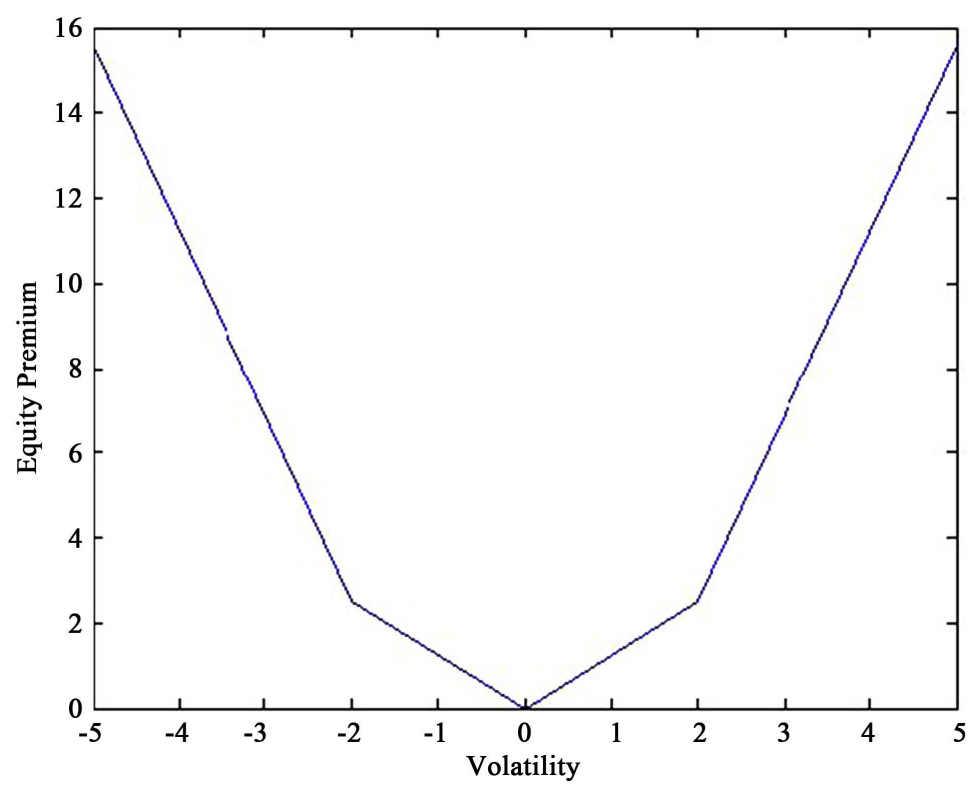

Figure 2. Power utility volatility effect under binomial distribution when no jump is expected.

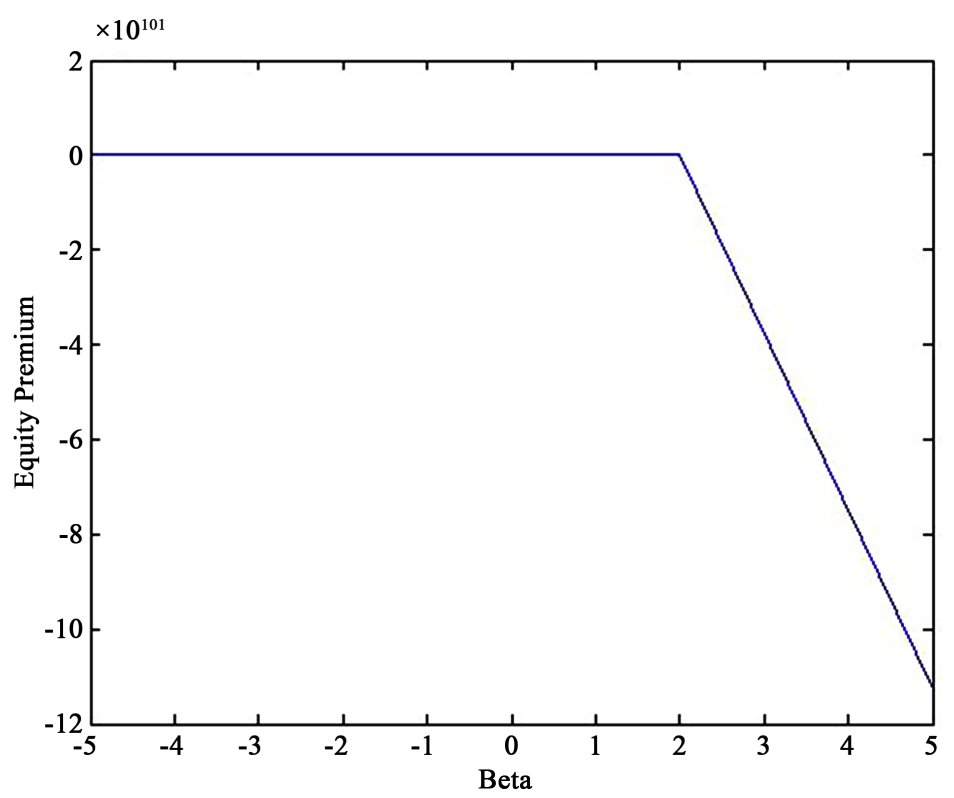

Figure 3. Power utility beta effect under binomial distribution.

Proof. For the square root utility function, the rare-event premium is given by

$$
\begin{aligned}
\phi_{N}=\lambda E\left[\left(\mathrm{e}^{x}-1\right)\left(1-V_{t} \mathrm{e}^{-\frac{1}{2} x}\right)\right](\text { see }[10]) \\
\phi_{N}=\lambda E\left[\left(\mathrm{e}^{x}-1\right)\left(1-V_{t} \mathrm{e}^{-\frac{1}{2} x}\right)\right]=\lambda E\left[\mathrm{e}^{x}-V_{t} \mathrm{e}^{\frac{1}{2} x}-1+V_{t} \mathrm{e}^{-\frac{1}{2} x}\right] \\
=\lambda\left[E\left(\mathrm{e}^{x}\right)-q E\left(\mathrm{e}^{\frac{1}{2} x}\right)-E(1)+q E\left(\mathrm{e}^{-\frac{1}{2} x}\right)\right]
\end{aligned}
$$




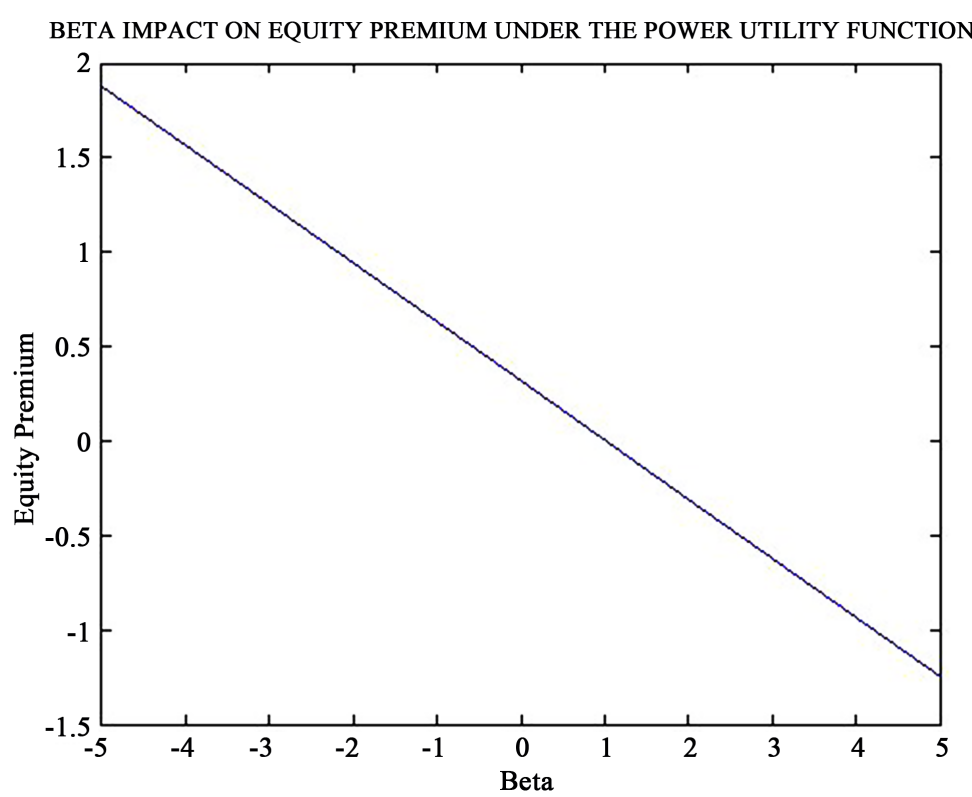

Figure 4. Power utility beta effect under binomial distribution when no jump is expected.

Since $x \sim B(n, p)$, we have that

$$
E\left[\mathrm{e}^{X}\right]=(1-p+p \mathrm{e})^{n}=(1+(\mathrm{e}-1) p)^{n}=m_{X}(1)
$$

and

$$
E\left[\mathrm{e}^{\frac{1}{2} X}\right]=\left(1-p+p \mathrm{e}^{\frac{1}{2}}\right)^{n}=m_{X}\left(\frac{1}{2}\right)
$$

Also

$$
E\left[\mathrm{e}^{-\frac{1}{2} X}\right]=\left(1-p+p \mathrm{e}^{-\frac{1}{2}}\right)^{n}=m_{X}\left(-\frac{1}{2}\right)
$$

Thus our rare-event premium is

$$
\lambda\left[(1+(\mathrm{e}-1) p)^{n}-q\left(1-p+p \mathrm{e}^{\frac{1}{2}}\right)^{n}-1+q\left(1-p+p \mathrm{e}^{-\frac{1}{2}}\right)^{n}\right]
$$

and therefore our equity premium is

$$
\begin{aligned}
\phi= & \rho X_{0}(t)-\rho+\frac{\delta^{2}}{2 V_{t}}+\lambda(1+(\mathrm{e}-1) p)^{n}-\lambda q\left(1-p+p \mathrm{e}^{\frac{1}{2}}\right)^{n} \\
& -\lambda+\lambda q\left(1-p+p \mathrm{e}^{-\frac{1}{2}}\right)^{n}
\end{aligned}
$$

We observe a constant premium of 1.722 in Figure 5 regardless of volatility in the process for square root utility. What is important here is that, the premium factor is positive indicating a fair compensation on the investment. However, Figure 6 is consistent with Figure 2 when no jumps are expected. These two figures confirm the positive judgement that this premium posses in the semi 


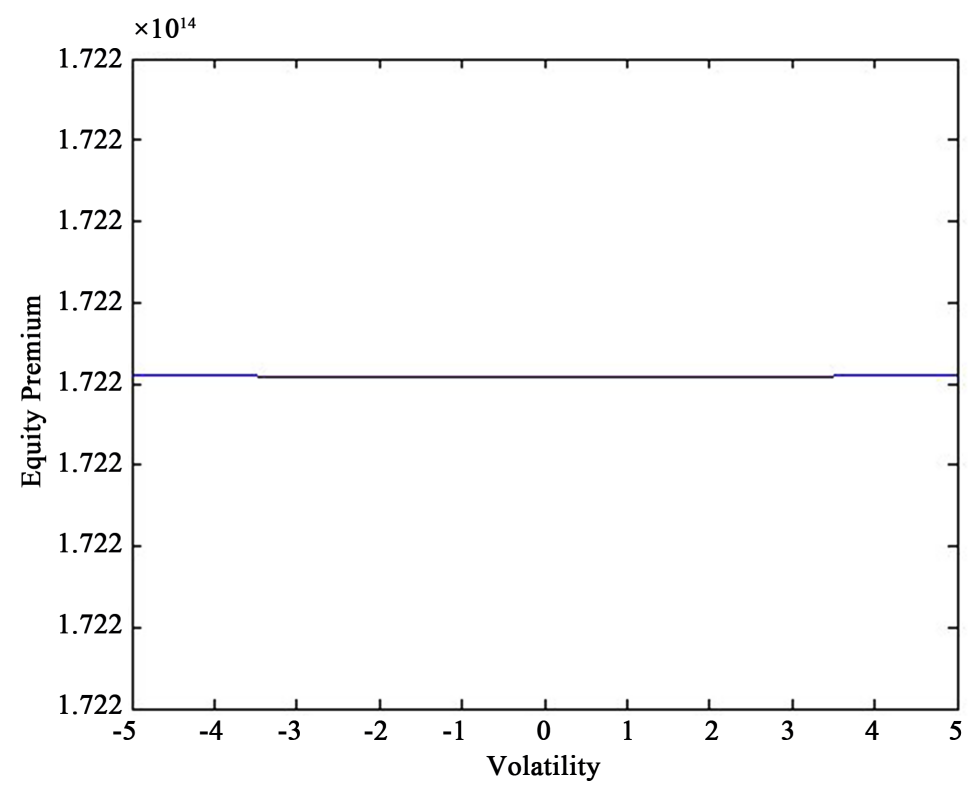

Figure 5. Square root utility volatility effect under binomial distribution.

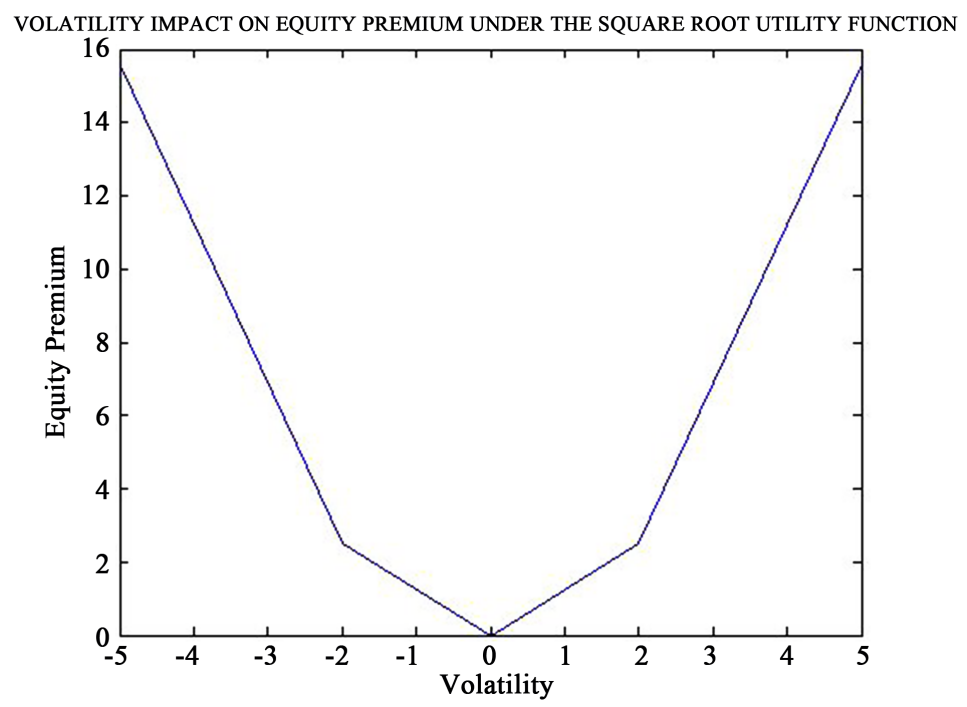

Figure 6. Square root utility volatility effect under binomial distribution when no jump is expected.

martingale market. Investors should therefore take advantage and consider investing in a market like this one.

In practice, the square root utility has many advantages in finance and economics including it's ability to minimize shocks in the stock market. We were also able to see that the results for this utility function in terms of equilibrium equity premium were significantly reasonable compared to other utility functions in the martingale market.

Theorem 3. An investor's equilibrium equity premium with quadratic utility function $U\left(r_{t}\right)=r_{t}-a r_{t}^{2}, a>0$ in the semi martingale market with normal jumps is given by 


$$
\begin{aligned}
\phi= & \rho X_{0}(t)-\rho+\frac{2 a \delta^{2}}{1-2 a V_{t}}+\lambda\left[(1+(\mathrm{e}-1) p)^{n}-\frac{q(1+(\mathrm{e}-1) p)^{n}}{1-2 a q}\right. \\
& \left.+\frac{2 a q^{2}\left(1-p+p \mathrm{e}^{2}\right)^{n}}{1-2 a q}-1+\frac{q}{1-2 a q}-\frac{2 a q^{2}(1+(\mathrm{e}-1) p)^{n}}{1-2 a q}\right]
\end{aligned}
$$

where $\phi_{\delta}=\rho X_{0}(t)-\rho+\frac{2 a \delta^{2}}{1-2 a V_{t}}$ is the diffusive risk premium and

$$
\begin{aligned}
\phi_{N}=\lambda & {\left[(1+(\mathrm{e}-1) p)^{n}-\frac{q(1+(\mathrm{e}-1) p)^{n}}{1-2 a q}+\frac{2 a q^{2}\left(1-p+p \mathrm{e}^{2}\right)^{n}}{1-2 a q}\right.} \\
& \left.-1+\frac{q}{1-2 a q}-\frac{2 a q^{2}(1+(\mathrm{e}-1) p)^{n}}{1-2 a q}\right]
\end{aligned}
$$

is the rare-event

premium.

Proof. For the HARA Quadratic utility function,

$$
\begin{aligned}
\phi_{N}=\lambda E\left[\left(\mathrm{e}^{x}-1\right)\left(1-\frac{V_{t}\left(1-2 a V_{t} \mathrm{e}^{x}\right)}{1-2 a V_{t}}\right)\right] \text { so that } \\
\phi_{N}=\lambda E\left[\left(\mathrm{e}^{x}-1\right)\left(1-\frac{V_{t}\left(1-2 a V_{t} \mathrm{e}^{x}\right)}{1-2 a V_{t}}\right)\right] \\
=\lambda E\left[\mathrm{e}^{x}-\frac{V_{t} \mathrm{e}^{x}-2 a V_{t}^{2} \mathrm{e}^{2 x}}{1-2 a V_{t}}-1+\frac{V_{t}}{1-2 a V_{t}}-\frac{2 a V_{t}^{2} \mathrm{e}^{x}}{1-2 a V_{t}}\right] \\
=\lambda E\left[\mathrm{e}^{x}-\frac{V_{t} \mathrm{e}^{x}}{1-2 a V_{t}}+\frac{2 a V_{t}^{2} \mathrm{e}^{2 x}}{1-2 a V_{t}}-1+\frac{V_{t}}{1-2 a V_{t}}-\frac{2 a V_{t}^{2} \mathrm{e}^{x}}{1-2 a V_{t}}\right] \\
=\lambda\left[E\left(\mathrm{e}^{x}\right)-\frac{q E\left(\mathrm{e}^{x}\right)}{1-2 a q}+\frac{2 a q^{2} E\left(\mathrm{e}^{2 x}\right)}{1-2 a q}-1+\frac{q}{1-2 a q}-\frac{2 a q^{2} E\left(\mathrm{e}^{x}\right)}{1-2 a q}\right]
\end{aligned}
$$

Now since $x \sim B(n, p)$, we have that

$$
E\left[\mathrm{e}^{X}\right]=(1-p+p \mathrm{e})^{n}=(1+(\mathrm{e}-1) p)^{n}=m_{X}(1)
$$

and

$$
E\left[\mathrm{e}^{2 X}\right]=\left(1-p+p \mathrm{e}^{2}\right)^{n}=m_{X}(2) .
$$

thus our rare-event premium is

$$
\begin{aligned}
& \lambda\left[(1+(\mathrm{e}-1) p)^{n}-\frac{q(1+(\mathrm{e}-1) p)^{n}}{1-2 a q}+\frac{2 a q^{2}\left(1-p+p \mathrm{e}^{2}\right)^{n}}{1-2 a q}\right. \\
& \left.-1+\frac{q}{1-2 a q}-\frac{2 a q^{2}(1+(\mathrm{e}-1) p)^{n}}{1-2 a q}\right]
\end{aligned}
$$

which implies that our equity premium is 


$$
\begin{aligned}
\phi= & \rho X_{0}(t)-\rho+\frac{2 a \delta^{2}}{1-2 a V_{t}}+\lambda\left[(1+(\mathrm{e}-1) p)^{n}-\frac{q(1+(\mathrm{e}-1) p)^{n}}{1-2 a q}\right. \\
& \left.+\frac{2 a q^{2}\left(1-p+p \mathrm{e}^{2}\right)^{n}}{1-2 a q}-1+\frac{q}{1-2 a q}-\frac{2 a q^{2}(1+(\mathrm{e}-1) p)^{n}}{1-2 a q}\right]
\end{aligned}
$$

The results in Figure 7 and Figure 8 are similar to those obtained under the power utility except that, the quadratic utility is affected by the wealth process $V_{t}$. It is clear in Figure 9 that the equilibrium equity premium is constant regardless of the wealth value. However, under the quadratic utility function, the investor always recieves a fair compensation for having taken some risk as long as no jump is expected. This is evident in Figure 10.

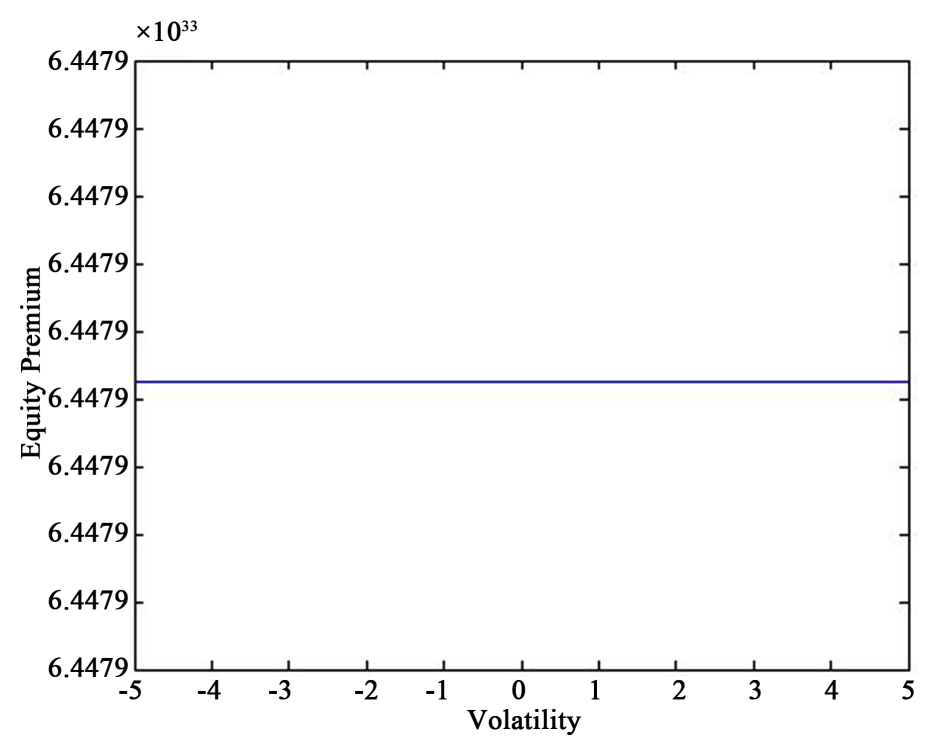

Figure 7. Quadratic utility volatility effect under binomial distribution.

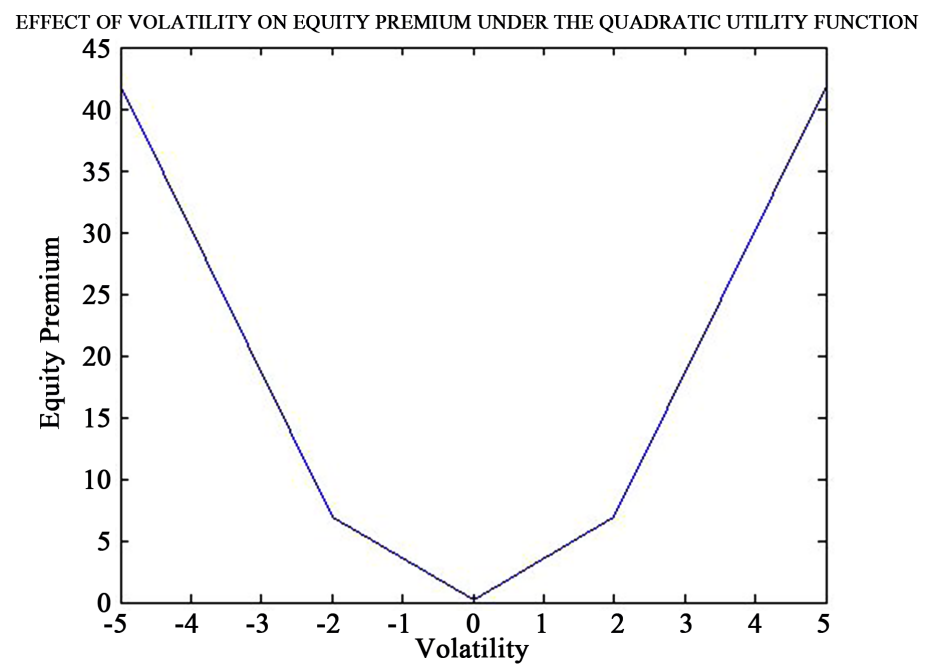

Figure 8. Quadratic utility volatility effect under binomial distribution when no jump is expected. 


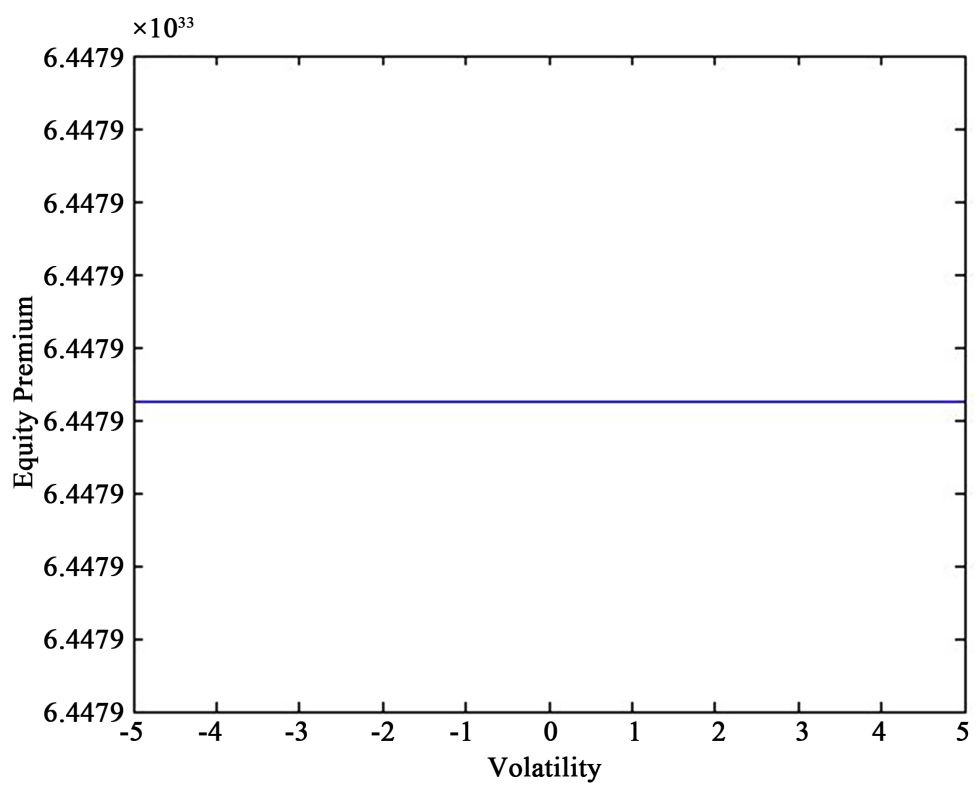

Figure 9. Quadratic utility wealth effect under binomial distribution.

EFFECT OF V(t) ON EQUITY PREMIUM UNDER THE QUADRATIC UTILITY FUNCTION

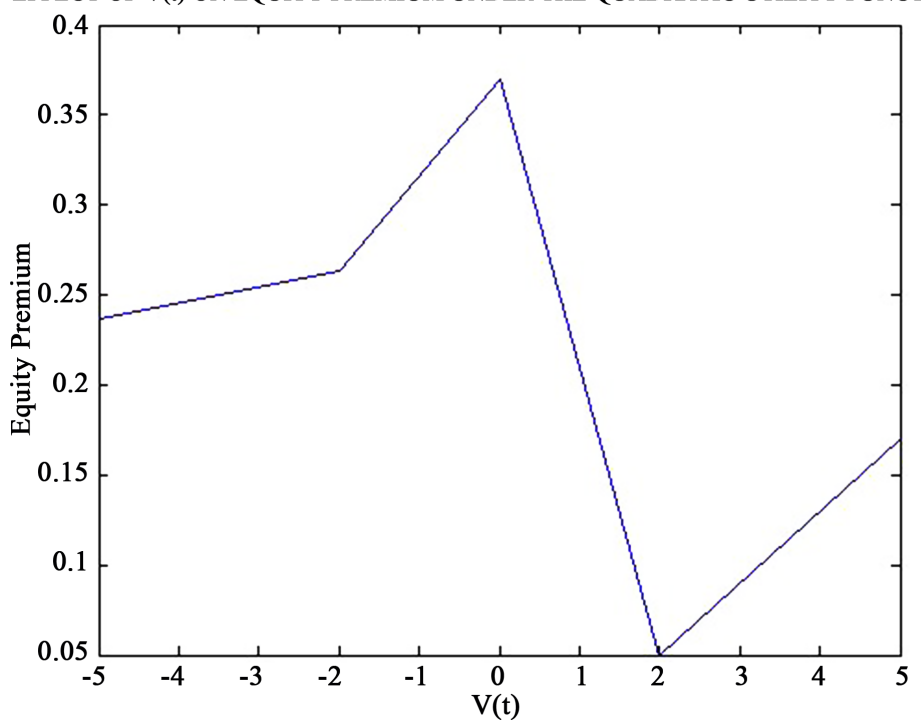

Figure 10. Quadratic utility wealth effect under binomial distribution when no jump is expected.

All in all, it is important to note that the real life processes are not martingales but prices are normalized so that the processes can then be martingales. This means that jumps must be expected in any normalized market although most scholars have generally assumed the processes without jumps. In comparison, the results in the martingale market are similar to those of the semi martingale market but the premium is not as attractive as the one realised from the semi martingale market. Infact, in this market, the equity premium is always positive regarless of the utility function the investor is following. We therefore urge investors to consider investing in this market. 


\section{Conclusions and Suggestions}

The martingale and semi martingale markets differ significantly in terms of how much compensation an investor recieves for having taken some risk in the investment. This is the case whenever jump amplitudes follow a binomial distribution in a semi martingale market. We observe consistent results in the equity premium of the power, square root and quadratic utility functions in terms of volatility effect, but the quadratic utility is affected also by the wealth process $V_{t}$. We therefore advise investors consuming quadratically to consider investing in the semi martingale market with jumps as long as the amplitudes follow a binomial distribution. This is to avoid external shocks and variance in the premium when jumps are not expected.

\section{Acknowledgements}

We thank the editor and the referee for their comments.

\section{Conflicts of Interest}

The authors declare no conflicts of interest regarding the publication of this paper.

\section{References}

[1] Mukupa, G.M. and Offen, E.R. (2015) The Impact of Utility Functions on the Equilibrium Equity Premium in a Production Economy with Jump Diffusion. IAENG International Journal of Applied Mathematics, 45, 120-127.

[2] George, M.M. and Offen, E.R. (2016) Equity Premium under Normally Distributed Jump Sizes in a Production Economy with Jumps. International Journal of Applied Mathematics and Statistics, 54, 27-41.

[3] Zhang, J.E., Zhao, H.M. and Chang, E.C. (2012) Equilibrium Asset and Option Pricing under Jump Diffusion. The University of Hong Kong, Mathematical Finance, 22, 538-568. https://doi.org/10.1111/j.1467-9965.2010.00468.x

[4] Wissem, B., Anand, N.P. and Faouzi, T. (2011) Pricing and Hedging of Asian Option under Jumps. IAENG International Journal of Applied Mathematics, 41, 310-319.

[5] Sidorov, S.P., et al. (2014) Stock Volatility Modelling with Augmented GARCH Model with Jumps. IAENG International Journal of Applied Mathematics, 44, 212-220.

[6] Frontczak, R. (2013) Pricing Options in Jump Diffusion Models Using Mellin Transforms. Journal for Mathematical Finance (JMF), 3, 366-373. https://doi.org/10.4236/jmf.2013.33037

[7] Makate, N. and Sattayatham, P. (2011) Stochastic Volatility Jump-Diffusion Model for Option Pricing. Journal of Mathematical Finance, 1, 90-97. https://doi.org/10.4236/jmf.2011.13012

[8] Ross, S.M. (2003) An Elementary Introduction to Mathematical Finance: Options and Other Topics. University Cambridge Press, New York.

[9] Mukupa, G.M., Offen, E.R., Lungu, E.M. and Kunda, D. (2016) A Comparative Study of Equilibrium Equity Premium under Discrete Distributions of Jump Am- 
plitudes. Journal for Mathematical Finance (JMF), 6, 232-246.

https://doi.org/10.4236/jmf.2016.61020

[10] Mukupa, G.M., Offen, E.R. and Lungu, E.M. (2016) The Risk Averse Investor's Equilibrium Equity Premium in a Semi Martingale Market with Arbitrary Jumps. Journal of Mathematics Research, 8, 139-147. https://doi.org/10.5539/jmr.v8n6p139

[11] Amin, K.I. (1993) Jump Diffusion Option Valuation in Discrete Time. The Journal of Finance, 48, 1833-1863. https://doi.org/10.1111/j.1540-6261.1993.tb05130.x

[12] Lewis, A.L. (2001) A Simple Option Formula For General Jump-Diffusion and Other Exponential Lévy Processes. Envision Financial Systems and Option City.

[13] Kou, S.G. (2002) A Jump-Diffusion Model for Option Pricing. Department of Industrial Engineering and Operations Research, Columbia University, New York.

[14] Lyuu, Y.-D. (2002) Financial Engineering and Computation; Principles, Mathematics, Algorithms. Cambridge University Press, United Kingdom.

[15] Liu, J., Pan, J. and Wang, T. (2005) An Equilibrium Model of Rare-Event Premium and Its Implication for Option Smirks. The Review of Financial Studies, 18, 131-164. https://doi.org/10.1093/rfs/hhi011

[16] Bates, D.S. (2008) The Market for Crash Risk. Journal of Economic Dynamics and Control, 32, 2291-2321. https://doi.org/10.1016/j.jedc.2007.09.020

[17] Santa-Clara, P. and Yan, S. (2010) Crashes, Volatility, and the Equity Premium: Lessons from S and P 500 Options.

[18] El Karoui, Jeanblanc-Picquè, M. and Shreve, S.E. (1998) Robustness of the Black and Scholes Formula. Mathematical Finance, 8, 93-126. https://doi.org/10.1111/1467-9965.00047

[19] Hobson, D.G. (1998) Volatility Misspecification, Option Pricing and Superreplication via Coupling. The Annals of Applied Probability, 8, 193-205. https://doi.org/10.1214/aoap/1027961040

[20] Bellamy, N. and Jeanblanc, M. (2000) Incompleteness of Markets Driven by a Mixed Diffusion. Finance and Stochastics, 4, 209-222. https://doi.org/10.1007/s007800050012

[21] Henderson, V. (2002) Analytical Comparisons of Option Prices in Stochastic Volatility Models. Oxford Financial Research Centre Preprint 2002-MF-03.

[22] Henderson, V. and Hobson, D.G. (2003) Coupling and Option Price Comparisons in a Jump-Diffusion Model. Stochastics and Stochastics Reports, 75, 79-101.

[23] Henderson, V., et al. (2003) A Comparison of q-Optimal Option Prices in a Stochastic Volatility Model with Correlation. Oxford Financial Research Centre Preprint 2003-MF-02.

[24] Møller, T. (2003) Stochastic Orders in Dynamic Reinsurance Markets.

[25] Eberlein, E. and Jacod, J. (1997) On the Range of Options Prices. Finance and Stochastics, 1, 131-140. https://doi.org/10.1007/s007800050019

[26] Frey, R. and Sin, C.A. (1999) Bounds on European Option Prices under Stochastic Volatility. Mathematical Finance, 9, 97-116. https://doi.org/10.1111/1467-9965.00064

[27] Jakubenas, P. (2002) On Option Pricing in Certain Incomplete Markets. Steklov Institute of Mathematics, Moscow, 114-133.

[28] Gushchin, A.A. and Mordecki, E. (2002) Bounds on Option Prices for Semimartingale Market Models. Proceedings of the Steklov Institute of Mathematics, 237, 73-113. 